OPEN ACCESS

Edited by:

Rentian Feng,

University of Pittsburgh,

United States

Reviewed by:

Luiz Cunha,

Federal University of Pará, Brazil

Luis E. Arias-Romero,

National Autonomous University of

Mexico, Mexico

*Correspondence:

Zhixiang Zhuang

zzxf163@163.com

Chunliang Liu

Icl1986@suda.edu.cn

${ }^{\dagger}$ These authors have contributed equally to this work and share first authorship

Specialty section:

This article was submitted to Cancer Molecular Targets and Therapeutics, a section of the journal

Frontiers in Oncology

Received: 15 March 2021 Accepted: 01 June 2021

Published: 23 June 2021

Citation:

Feng F, Liu C, Bian H, Cai W, Zhou Y, Zhou $L$ and Zhuang Z (2021) TIPE2

Suppresses Malignancy of Pancreatic

Cancer Through Inhibiting TGF $\beta 1$ Mediated Signaling Pathway.

Front. Oncol. 11:680985.

doi: 10.3389/fonc.2021.680985

\section{TIPE2 Suppresses Malignancy of Pancreatic Cancer Through Inhibiting TGFß1 Mediated Signaling Pathway}

\author{
Fang Feng ${ }^{1,2 \dagger}$, Chunliang Liu ${ }^{3 *+}$, Huahui Bian ${ }^{1}$, Wei Cai ${ }^{2}$, Ying Zhou ${ }^{2}$, Li Zhou ${ }^{2}$ \\ and Zhixiang Zhuang ${ }^{1 *}$ \\ ${ }^{1}$ Department of Oncology, The Second Affiliated Hospital of Soochow University, Medical College, Soochow University, \\ Suzhou, China, 2 Department of Oncology, Suzhou Ninth People's Hospital, Soochow University, Suzhou, China, \\ 3 Jiangsu Institute of Hematology, The First Affiliated Hospital of Soochow University, Suzhou, China
}

Pancreatic cancer is one of the major reasons of cancer-associated deaths due to poor diagnosis, high metastasis and drug resistance. Therefore, it is important to understand the cellular and molecular mechanisms of pancreatic cancer to identify new targets for the treatment. TIPE2 is an essential regulator of tumor apoptosis, inflammation and immune homeostasis. However, the role of TIPE2 is still not fully understood in pancreatic cancer. In this study, we found the expression of TIPE2 was decreased in pancreatic cancer tissues compare to paracancerous tissues, which was negatively correlated with tumor size in patients. Overexpression of TIPE2 significantly decreased cell proliferation, metastasis and increased apoptotic events in pancreatic cancer cell lines. Moreover, the results obtained from real time PCR and western blot revealed that TIPE2 was also involved in inhibiting MMPs and $\mathrm{N}$-Cadherin expression while increasing Bax expression in pancreatic cancer cells. Similarly, TIPE2 could inhibit tumor growth in vivo, decrease the expression of Ki-67 and $\mathrm{N}$-Cadherin, and increase the expression of Bax by $\mathrm{IHC}$ analysis in tumor tissues isolated from tumor-bearing mice. Mechanistic studies exhibited that TIPE2 might suppress pancreatic cancer development through inhibiting PI3K/AKT and Raf/MEK/ERK signaling pathways triggered by TGF $\beta 1$. Moreover, the tumor-infiltrating lymphocytes from tumor-bearing mice were analyzed by flow cytometry, and showed that TIPE2 could promote $T$ cell activation to exert an anti-tumor effect possibly through activation of DCs in a TGF $\beta 1$ dependent manner. In general, we described the multiple regulatory mechanisms of TIPE2 in pancreatic tumorigenesis and tumor microenvironment, which suggested TIPE2 may act as a potential therapeutic target in pancreatic cancer.

Keywords: TIPE2, pancreatic cancer, malignancy, TGF $\beta 1$, tumor microenvironment 


\section{INTRODUCTION}

Pancreatic cancer is one of the major causes of cancer-related mortality with a mournful 5-year survival rate of $9 \%$ (1). By the next decade, it is estimated that pancreatic cancer will rise to the second leading cause of cancer related death bypassing breast and colon cancer (2). About $90 \%$ of pancreatic cancer is pancreatic ductal adenocarcinoma, the treatment of pancreatic cancer is mainly based on comprehensive surgical intervention. However, the surgical success rate is low and the postoperative recurrence rate is high. For many years, the current standard of care for advanced pancreatic cancer is gemcitabine-based regimens (3), with an effective rate lower than $10 \%$, and its long-term use usually leads to drug resistance (4). Even the immune checkpoint blockades, including programmed death-1/ programmed death-ligand 1 (PD-1/PD-L1), and cytotoxic $\mathrm{T}$ lymphocyte associated antigen-4 (CTLA-4), which have been demonstrated robust results in melanoma, lung cancer, renal cell carcinoma, urothelial cancer, head and neck cancer and other malignancies (5), but most phase I and II clinical trials have failed to show any clinical efficacy in "cold tumor" pancreatic cancer (6).

As we know, pancreatic cancer patients are usually diagnosed after metastasis and/or significant local invasion have occurred, which dramatically decrease the survival rate (7). Moreover, the immunosuppressive tumor microenvironment in pancreatic cancer is another important factor, which can promote oncogenic progression (8). Therefore, it is necessary to know the mechanisms such as regulation of apoptotic signal transduction, metastasis and tumor microenvironment to identify new promising targets that may help to enable immune mediated control of this disease.

TIPE2 (tumor necrosis factor- $\alpha$ induced protein 8 -like 2, or TNFAIP8L2), is a member of the TIPE family that is preferentially expressed in lymphoid and inflamed tissues. TIPE2 is an essential regulator of apoptosis, inflammation and immune homeostasis (9, 10). Overexpression of TIPE2 in tumor cells induced cell apoptosis and significantly inhibited Ras-induced tumorigenesis through binding to Ras-interacting domain of RGL and inhibiting the activation of Ral (9). In addition, TIPE2 inhibited invasiveness and tumor development via reducing MMP9 expression by targeting Rac1 in hepatocellular carcinoma (HCC) and gastrointestinal stromal tumor $(11,12)$. TIPE2 also inhibited HCC metastasis through suppressing Erk1/2 and NF- $\mathrm{KB}$ activation (13). Furthermore, TIPE2 suppressed the epithelial-mesenchymal transition (EMT) and metastasis of tumor cells via suppressing PI3K/Akt and Wnt/B-catenin signaling pathways in some tumor types, including glioma, gastric cancer and breast cancer (14-16). Moreover, TIPE2 could bind to $\beta$-catenin directly to decrease the occurrence of EMT and suppress metastasis of endometrial cells (17). As an important regulator of tumor microenvironment, TIPE2 was found to significantly inhibit oncogenic progression through activating $\mathrm{T}$ and $\mathrm{NK}$ cells, and inhibiting FoxP3 ${ }^{+}$Treg cells in tumor microenvironment $(18,19)$.

In this manner, TIPE2 acts as an important regulator in tumor development and tumor microenvironment during oncogenic progression. However, whether TIPE2 is involved in pancreatic cancer is still not fully understood. In this study, we describe the multiple regulatory mechanisms in pancreatic cancer development and tumor microenvironment.

\section{MATERIALS AND METHODS}

\section{Experimental Animals}

Specific pathogen-free female nude mice (6-8 weeks) and wild type C57BL/6J (6-8 weeks) mice were purchased from Vital River Laboratory Animal Center (Beijing, China). All mice were maintained in specific pathogen-free facilities in accordance with the National Animal Care and Use Committee. The animal studies were approved by the Institutional Laboratory Animal Care and Use Committee of Soochow University.

\section{Immunohistochemistry}

Pancreatic cancer and adjacent tissue microarray (70 cases, Outdo Biotech, Shanghai) was stained with rabbit anti-TIPE2 antibody (ProteinTech, Chicago, IL) and HRP-conjugated anti-rabbit IgG secondary antibody (Cell Signaling Technology, Danvers, MA). The immunohistochemical staining results were scored by considering the intensity of staining and the percentage of positive cells. The intensity of staining was scored as follows: 0 , negative; 1 , weak; 2 , moderate; 3 , strong. The percentage of positive stained cells was defined as follows: $0,<5 \% ; 1,6-25 \% ; 2$, $26-50 \% ; 3,51-75 \% ; 4,76-100 \%$. The intensity of staining multiplies the percentage of positive cells to produce a final score of TIPE2 expression as follows: 0 , total score $=0 ; 1+$, total score $=1-4 ; 2+$, total score $=5-8 ; 3+$, total score $=9-12$.

Tumor tissues isolated from mice were fixed in $10 \%$ neutral buffered formalin and embedded in paraffin. Slides were stained with primary antibody, including anti-TIPE2 (ProteinTech), antiKi67 (Abcam, Cambridge, MA), anti-TGFß1 (ProteinTech), antiBax (Cell Signaling Technology) and anti-N-cadherin (Cell Signaling Technology), and then stained with HRP anti-rabbit IgG secondary antibody (Cell Signaling Technology).

\section{Cell Culture}

AsPC-1, BxPC-3 and 293T cell lines were purchased from the Cell Bank of Chinese Scientific Academy (Shanghai, China), Panc02 cell line was purchased from the National Experimental Cell Resource Sharing platform (Beijing, China). No mycoplasma contamination was detected in these cell lines by PCR analysis. AsPC-1 and BxPC-3 cells were cultured in 10\% FBS RPMI-1640. Panc02 and 293T cells were cultured in 10\% FBS DMEM.

In AsPC- 1 cells, the neutralizing anti-TGF $\beta 1$ monoclonal antibody ( $1 \mu \mathrm{g} / \mathrm{ml}$, ProteinTech) or $5 \mathrm{ng} / \mathrm{ml}$ human recombinant TGF $\beta 1$ (ProteinTech) was added for $48 \mathrm{~h}$ incubation, respectively.

\section{Overexpression of TIPE2 in Pancreatic Cancer Cell Lines}

The full coding sequences of human and murine TIPE2 were amplified and subcloned into lentiviral vector pCAG. The lentivirus encoding TIPE2 was produced by co-transfecting 293T cells with pCAG and lentivirus packaging plasmids. Empty pCAG vector was used as vector control. 
To overexpress TIPE2 in pancreatic cancer cell lines (AsPC-1, BxPC-3 and Panc02), all cell lines were infected with TIPE2expressing lentivirus and vector control lentivirus. The pancreatic cancer cell lines stably expressing TIPE2 or vector control were constructed by sorting GFP positive cells using BD FACS flow cytometer (San Jose, CA), and the expression of TIPE2 was confirmed by western blot analysis.

\section{Cell Proliferation and Cell Apoptosis Assay}

For cell proliferation assay, the cell proliferation was examined using cell counting kit-8 (CCK-8, Dojindo, Kumamoto, Japan) as described by the manufacturer's protocol. Cells were seeded in 96-well plate at a destiny of 3,000 cells/well (AsPC-1 and BxPC-3 cell lines) and 1,000 cells/well (Panc02 cell line), and then cultured for $0,24,48$ and $72 \mathrm{~h}$. At the indicated time point, $10 \mu \mathrm{l}$ of CCK- 8 solution was added to each well and incubated for additional $3 \mathrm{~h}$. The optical density (OD) value was measured at $450 \mathrm{~nm}$ on a microplate reader (BioTek, Winooski, VT).

For cell apoptosis assay, cells were seeded in 6-well plate at a destiny of $2 \times 10^{5}$ cells/well (AsPC- 1 and BxPC- 3 cell lines) and $1 \times 10^{5}$ cells/well (Panc02 cell line). The cells were collected $48 \mathrm{~h}$ after incubation, then stained with Annexin V and 7-AAD (BD Biosciences, San Diego, CA), and were analyzed using flow cytometry.

\section{Migration and Invasion Assay}

The highly metastatic pancreatic cancer cell line, AsPC-1, was used for cell migration and invasion analysis using transwell chambers (Corning, New York, NY) with $8-\mu \mathrm{m}$ pore size membrane inserts in 24-well plate. And the membrane inserts were coated with matrigel (BD Biosciences) for invasion assay. Some $150 \mu \mathrm{l}$ cells $\left(1 \times 10^{5} / \mathrm{ml}\right)$ were resuspended in serum-free medium and seeded onto the upper chambers with or without matrigel. The bottom chambers were filled with $600 \mu \mathrm{l}$ RPMI1640 medium supplemented with $10 \%$ FBS. After $16 \mathrm{~h}$ in migration assay and $24 \mathrm{~h}$ in invasion assay, the cells passed through the membrane were fixed with methanol and stained with $0.1 \%$ crystal violet. The stained cells were photographed and counted under the microscope.

\section{Quantitative Real Time PCR Analysis}

Total RNA was extracted from cells and was reverse-transcribed into cDNA by reverse transcription kit (TaKaRa, Otsu, Japan). The relative gene expression was detected by real-time PCR performed using SYBR Green PCR master mixes (Thermo Fisher, Waltham, MA). GAPDH was used as the internal control. The primers used for these analyses are listed in Supplementary Table 1.

\section{Xenograft Tumor Model}

Human AsPC-1/vector and AsPC-1/TIPE2 cells, BxPC-3/vector and BxPC-3/TIPE2 cells $\left(1 \times 10^{6}\right.$ cells in $100 \mu$ PBS per mouse $)$ were injected subcutaneously into the right hind legs of BALB/c nude mice (6-8 weeks). After ten days, the mice were monitored weekly for tumor size using a caliper. The tumor volume was calculated according to the standard formula: volume $=0.5 \times$ length $\times$ width $^{2}$. The mice were sacrificed when tumor volume reached approximate $400 \mathrm{~mm}^{3}$, then the tumor tissues were isolated and photographed.

\section{Western Blot Analysis}

Total protein was extracted from cell lines lysed with RIPA buffer (Beyotime, Shanghai, China) in the presence of a protease inhibitor and phosphatase inhibitor (Selleck, Shanghai, China). The concentration of protein was determined by BCA kit (Beyotime). Protein was resolved by SDS-PAGE and then transferred to polyvinylidene difluoride (PVDF) membrane (Millipore, Burlington, MA). The membranes were blocked with $5 \%$ non-fat milk in TBST for $2 \mathrm{~h}$ at room temperature and then incubated with primary antibodies overnight at $4^{\circ} \mathrm{C}$. The primary antibodies include rabbit anti-TIPE2 (ProteinTech), anti-pERK, anti-ERK, anti-pAKT, anti-AKT, anti-Bax (Cell Signaling Technology), anti-GAPDH (ProteinTech), antipTGFBR1 and anti-TGFBR1 (Affinity, Cincinnati, OH). After washing, membranes were incubated with HRP-conjugated secondary antibody (goat anti-rabbit IgG or goat anti-mouse IgG, Cell Signaling Technology) for $1 \mathrm{~h}$ at room temperature. After washing, the protein bands were visualized with enhanced chemiluminescence (ECL) detection kit (Beyotime).

\section{ELISA Analysis}

AsPC-1/vector, AsPC-1/TIPE2 cells, Panc02/vector and Panc02/ TIPE2 cells were seeded in 6-well plate at a destiny of $2 \times 10^{5}$ and $1 \times 10^{5}$. The supernatants were collected after $48 \mathrm{~h}$ of incubation and the protein level of TGF $\beta 1$ was analyzed using human TGF $\beta 1$ ELISA kit (Proteintech) and mouse TGF $\beta 1$ ELISA kit (Biosensis, Thebarton, South Australia).

\section{Subcutaneous Tumor Model}

Murine Panc02/vector and Panc02/TIPE2 cells $\left(1 \times 10^{6}\right.$ cells in $100 \mu \mathrm{l}$ PBS per mouse) were injected subcutaneously into the right hind legs of female C57BL/6J mice. After one week, the mice were monitored weekly for tumor size. The tumor volume was calculated by the formula: volume $=0.5 \times$ length $\times$ width $^{2}$. The mice were sacrificed when tumor volume reached approximate $400 \mathrm{~mm}^{3}$, then the tumor tissues were isolated, weighted and photographed. Then tumor-infiltrating lymphocytes were isolated for flow cytometry analysis.

\section{Flow Cytometry Analysis}

For analysis of tumor-infiltrating lymphocytes (TILs), tumorinfiltrating cells were isolated from tumors. The antibodies used for FACS staining including anti-mouse $\mathrm{CD} 3 €, \mathrm{CD} 4, \mathrm{CD} 8 \alpha, \mathrm{CD} 69$, CD62L, CD44, CD11c, CD80 and PD-L1 were purchased from Biolegend (San Diego, CA). Flow cytometry analysis was performed using a Novocyte flow cytometer (Agilent, Carpinteria, CA).

\section{Dendritic Cell (DC) Culture In Vitro}

Bone marrow cells isolated from $\mathrm{C} 57 \mathrm{BL} / 6$ mice were culture in 6 well plates $\left(1 \times 10^{6} \mathrm{cells} / \mathrm{ml}, 4 \mathrm{ml} /\right.$ well $)$ in the presence of $20 \mathrm{ng} /$ $\mathrm{ml} \mathrm{IL}-4$ and $40 \mathrm{ng} / \mathrm{ml} \mathrm{GM-CSF}$. On day 2, change the cell culture medium fully and softly with fresh RPMI-1640 medium containing IL-4 and GM-CSF. On days 4 and 6, replace one half of culture medium with fresh complete RPMI-1640 medium. 
On day 7, the DCs were harvested and co-cultured with Panc02/ vector or Panc02/TIPE2 cells. After $24 \mathrm{~h}$, the cells were harvested and analyzed with flow cytometry.

\section{Statistical Analysis}

Results were presented as mean \pm SD. Statistical test was performed with student's $t$ test (unpaired, two-tailed) using Graphpad prism 5.0 software (Graphpad, San Diego, CA). For all analysis, $\mathrm{P}<0.05$ was considered to indicate a statistically significant difference.

\section{RESULTS}

\section{TIPE2 Expression Was Decreased in Human Pancreatic Carcinoma}

The expression of TIPE2 was analyzed in pancreatic carcinoma and paracancerous tissues using immunohistochemistry. The proportion and the staining intensity of TIPE2-positive cells were obviously lower in carcinoma than in paracancerous tissue (Figures 1A, B). To further investigate the clinical significance of TIPE2 expression in pancreatic cancer, we analyzed the relationship between TIPE2 expression and clinico-pathological features (Table 1). TIPE2 expression was negatively correlated with tumor size $(P=0.014)$, while there was no significant correlation between TIPE2 expression and age, gender, TNM stage or lymph node metastasis. These data suggested that TIPE2 might play an important role in the progression of pancreatic cancer.

\section{Overexpression of TIPE2 Inhibited the Proliferation of Pancreatic Cancer Cells and Increased Cell Apoptosis}

To evaluate the role of TIPE2 in pancreatic cancer, we overexpressed TIPE2 in pancreatic cancer cell lines with lentivirus, including human cell lines AsPC-1 and BxPC-3, and mouse cell line Panc02, which have low level of TIPE2 expression. And we demonstrated that TIPE2 was successfully overexpressed in these cell lines compared with vector controls by western blot analysis (Figure 2A). To assess the function of TIPE2 in tumor cell biological activity in vitro, the cell proliferation and cell apoptosis assays were performed. TIPE2 markedly inhibited cell proliferation of pancreatic cancer cells using CCK-8 assay (Figure 2B). TIPE2 expression also significantly increased cell apoptosis of the pancreatic cancer cells (Figure 2C).
A

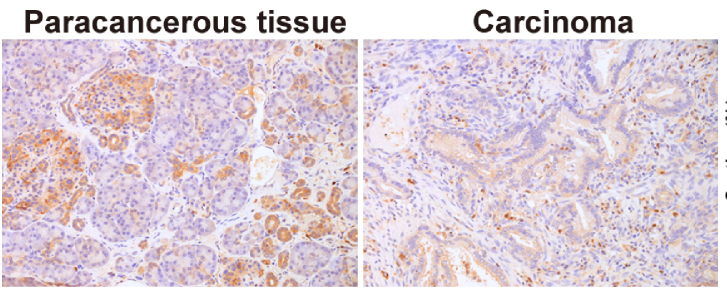

B

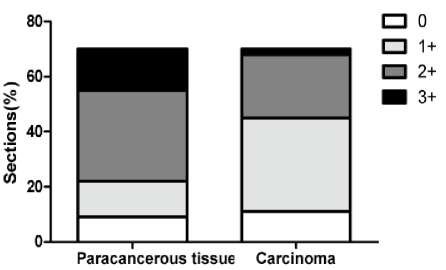

FIGURE 1 | The expression of TIPE2 in human pancreatic carcinoma tissues. (A) TIPE2 expression was examined in pancreatic carcinoma and paracancerous tissues with rabbit anti-TIPE2 antibody using immunohistochemistry. The positive cells were stained brown. Original magnification: 200x. (B) All tissues were scored by considering the intensity of staining and the percentage of positive cells.

TABLE 1 | Relationship between TIPE2 expression and clinico-pathological features in pancreatic cancer.

\begin{tabular}{|c|c|c|c|c|}
\hline Clinico-pathological features & Number & \multicolumn{2}{|c|}{ TIPE2 } & $P$ \\
\hline \multicolumn{5}{|l|}{ Age } \\
\hline$\geq 60$ & 43 & 27 & 16 & \\
\hline \multicolumn{5}{|l|}{ Gender } \\
\hline Male & 46 & 29 & 17 & 0.764 \\
\hline$<5 \mathrm{~cm}$ & 49 & 27 & 22 & 0.014 \\
\hline$\geq 5 \mathrm{~cm}$ & 21 & 18 & 3 & \\
\hline \multicolumn{5}{|l|}{ TNM Stage } \\
\hline $1+\|$ & 48 & 30 & 18 & 0.645 \\
\hline$I I I+I V$ & 22 & 15 & 7 & \\
\hline \multicolumn{5}{|l|}{ Lymph node metastasis } \\
\hline
\end{tabular}




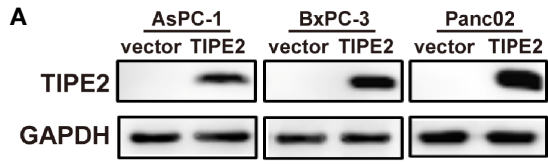

B
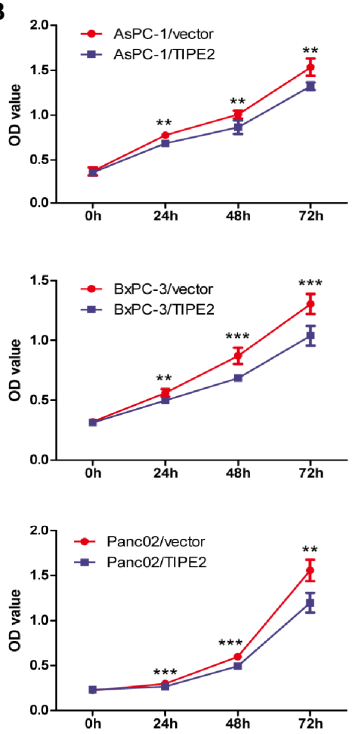

c

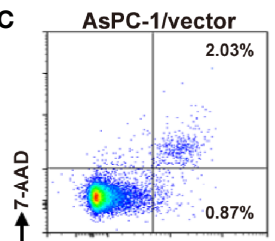

BxPC-3/vector

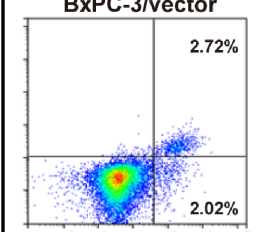

Panc02/vector

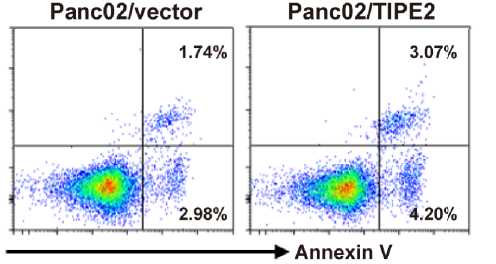

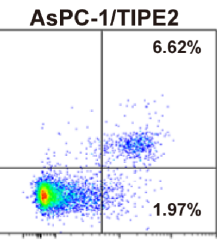

BXPC-3/TIPE2

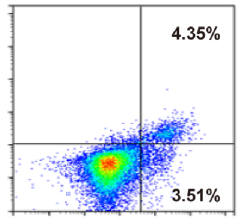

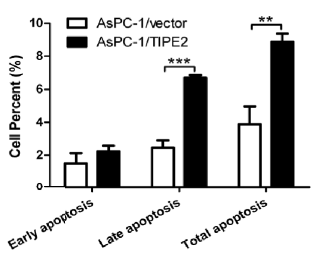
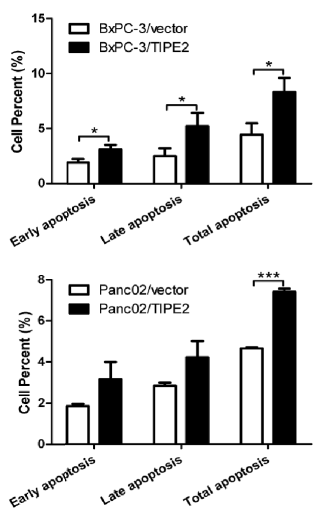

FIGURE 2 | Overexpression of TIPE2 inhibited cell proliferation and increased apoptosis of pancreatic cancer cells. (A) TIPE2-overexpressing or vector control cells were established by infecting with lentivirus and sorting with flow cytometry. The expression of TIPE2 was confirmed by western blot analysis. (B) Cell viability of TIPE2-overexpressing or vector control cells was examined using CCK-8. (C) Cells were stained with Annexin V and 7-AAD and analyzed by flow cytometry to detect the cell apoptosis (Annexin $\mathrm{V}^{+} / 7-\mathrm{AAD}^{-}$, early apoptosis: Annexin $\mathrm{V}^{+} / 7-\mathrm{AAD}^{+}$, late apoptosis). Data shown were representative of three independent experiments. Values are presented as means $\pm S D$. ${ }^{*} p<0.05,{ }^{* *} p<0.01,{ }^{* \star *} p<0.001$

\section{TIPE2 Suppressed the Metastasis of Pancreatic Cancer Cells In Vitro}

To investigate the function of TIPE2 in the migration and invasion of pancreatic cancer cells in vitro, we performed transwell assays using the highly metastatic pancreatic cancer cell line AsPC-1. TIPE2 overexpression could reduce the number of migrated and invaded cells (Figures 3A, B). The results showed that TIPE2 suppressed both the migration and invasion abilities of pancreatic cancer cells. To further explore the mechanism of TIPE2 in the metastasis of pancreatic cancer cells, we detected the expression of EMT markers, MMPs and Ncadherin. Overexpression of TIPE2 significantly reduced the expression of $M M P 1, M M P 2, M M P 3, M M P 9$ and $N$-cadherin (Figure 3C). Therefore, TIPE2 might suppress the metastasis of pancreatic cancer cells through inhibiting the EMT process.

\section{TIPE2 Suppressed Tumorigenesis of Pancreatic Cancer in Xenograft Tumor Models}

To explore the role of TIPE2 in pancreatic tumorigenesis in vivo, we established the subcutaneous xenograft tumor model using nude mice. The tumor volume was measured weekly from 10 days after tumor cell inoculation. TIPE2 significantly reduced the tumor volume compared with vector group during the progression of pancreatic cancer (Figures 4A, B). Then the tumors were isolated from mice which were sacrificed around 40 days after tumor cells injection. The tumor size of TIPE2 group was significantly smaller than that of vector group (Figures 4A, B). And we also confirmed TIPE2 was overexpressed in the pancreatic tumor tissues isolated from mice through immunohistochemical staining (Figure 4C). Moreover, TIPE2 overexpression could decrease the expression of Ki-67 and Ncadherin, and increase the expression of Bax (Figure 4C). These results showed that TIPE2 could affect tumor cell proliferation, apoptosis and EMT process, which demonstrated that TIPE2 could suppress the progression of pancreatic cancer in vivo.

\section{TIPE2 Inhibited PI3K/AKT and Raf/MEK/ ERK Signaling Pathways Triggered by TGF $\beta 1$}

Furthermore, we tried to peek into the mechanism of TIPE2 that mediates the function of pancreatic cancer cells. We detected the expression AKT, ERK and Bax which were involved in tumor proliferation, apoptosis, metastasis and EMT process. TIPE2 overexpression reduced the phosphorylation of ERK and AKT in all three cell lines (Figure 5A), and upregulated Bax expression in AsPC-1 and BxPC-3 cell lines (Figure 5A). As we know, TGF $\beta 1$ plays a key role in triggering PI3K/AKT and 

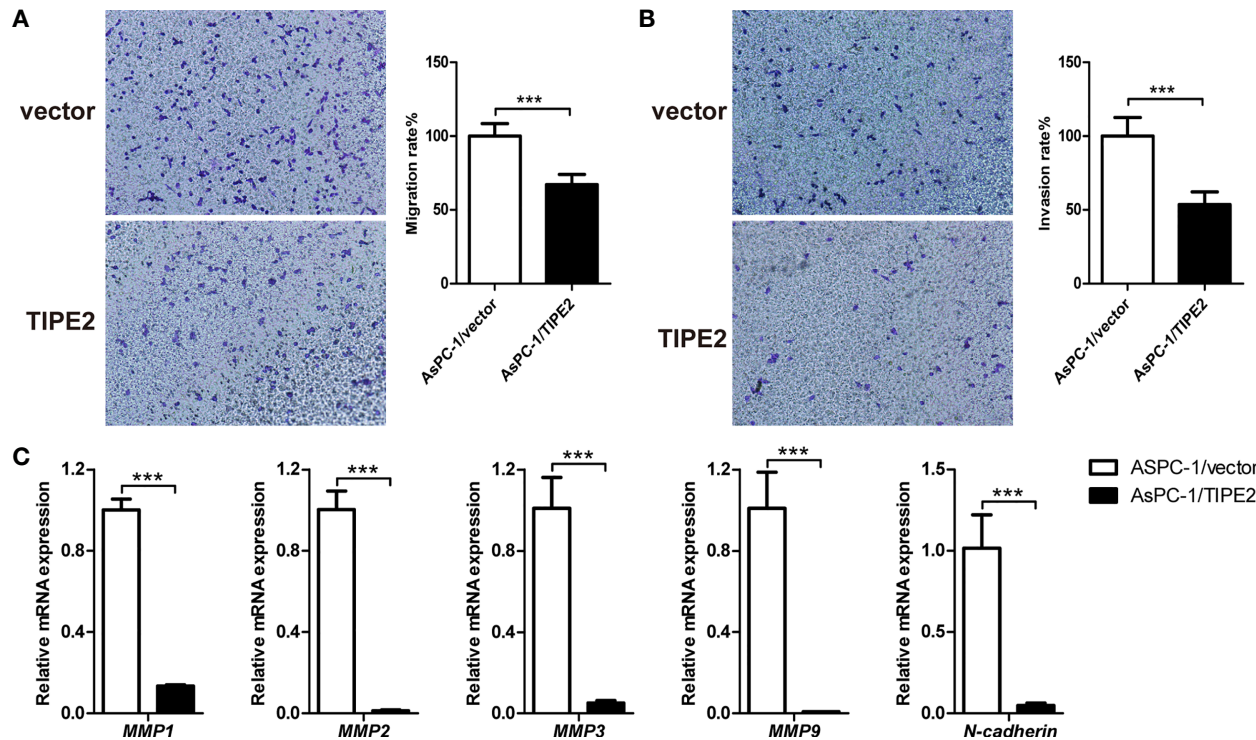

$\square$ ASPC-1/vector

AsPC-1/TIPE2

FIGURE 3 | TIPE2 suppressed the metastasis of pancreatic cancer cells in vitro. (A, B) Cell migration ability was determined with transwell assay, while cell invasion ability was determined with matrigel-coated transwell assay. The cells on the bottom side of the chamber were fixed, stained and counted. (C) Real-time gPCR analysis of MMP1, MMP2, MMP3, MMP9 and N-cadherin expression. The data shown are the representative of three independent experiments. Values are presented as means \pm SD. ${ }^{\star \star *} \mathrm{p}<0.001$.

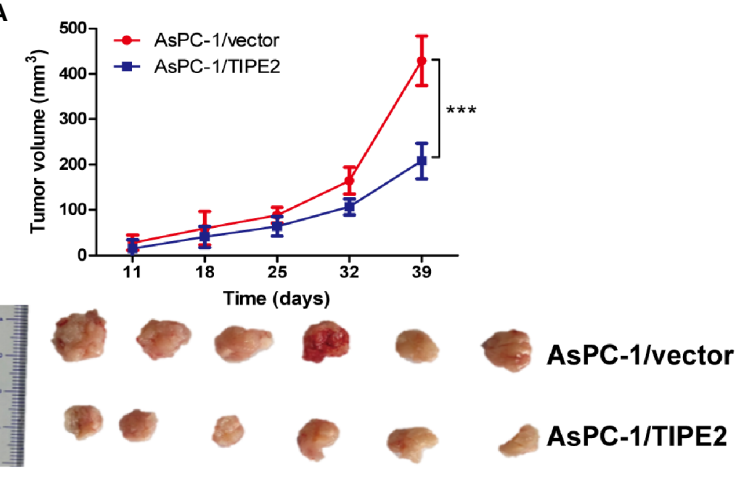

B

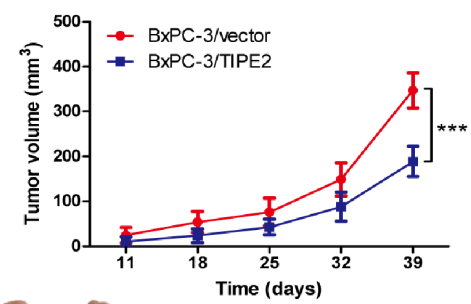

C.1

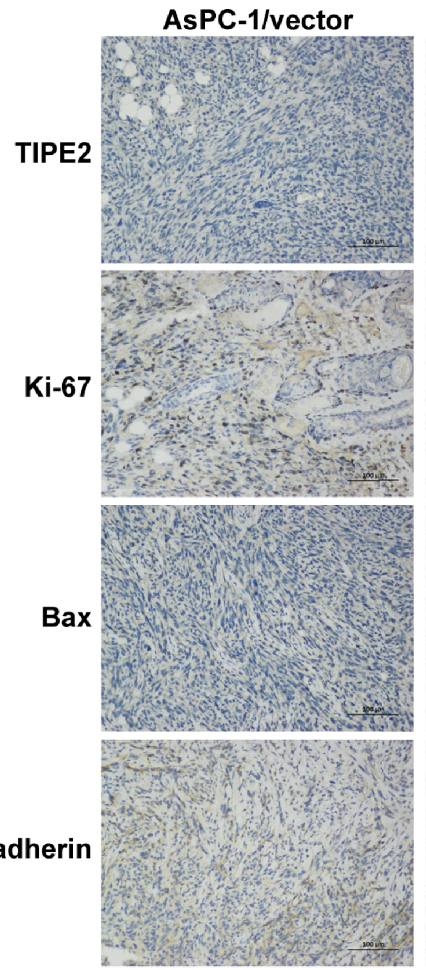

AsPC-1/TIPE2
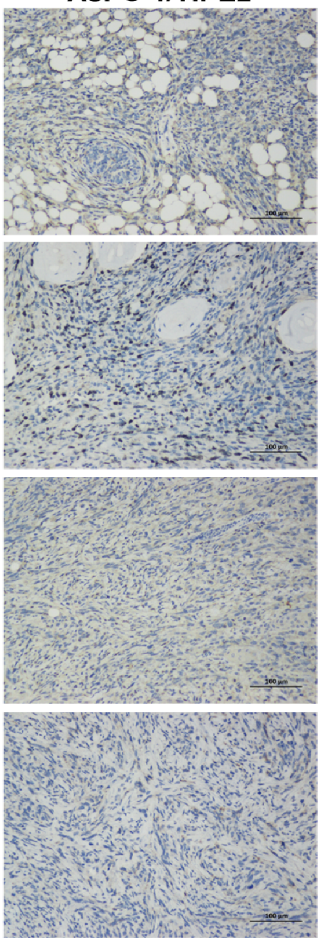

FIGURE 4 | TIPE2 suppressed the growth of pancreatic cancer in vivo. (A, B) The tumor volume was measured weekly ten days after tumor inoculation. Subcutaneous tumor growth curve was calculated. The tumors were isolated from nude mice and photographed 40 days after tumor cells inoculation. (C) TIPE2, $\mathrm{Ki}-67, \mathrm{Bax}$ and $\mathrm{N}$-cadherin expressions were examined in tumor tissues using immunohistochemistry. The data shown are the representative of three independent experiments. Values are presented as means $\pm \mathrm{SD} .{ }^{\star \star \star} \mathrm{p}<0.001$. 
Raf/MEK/ERK signaling pathways $(20,21)$, and we found that TIPE2 overexpression could reduce TGF $\beta 1$ secretion from AsPC-1 cells (Figure 5B), and similarly decreased the protein level of TGF $\beta 1$ in tumor tissues of tumor-bearing mice (Figure 5C). Moreover, the phosphorylation of TGFBR1 level was also reduced in TIPE2 overexpression cells (Figure 5D). Furthermore, we demonstrated that phosphorylation of ERK and AKT was decreased after blocking TGF $\beta 1$ with anti-TGF $\beta 1$ antibody and increased after incubated with rhTGF $\beta 1$ protein in AsPC-1 cells (Figure 5E). These results suggested TIPE2 might affect pancreatic cancer cells via inhibiting PI3K/AKT and Raf/MEK/ERK signaling pathways triggered by TGF $\beta 1$.

\section{TIPE2 Suppressed the Growth of Pancreatic Cancer Through Inhibiting TGF $\beta 1$ Expression in Subcutaneous Tumor Model}

TIPE2 can regulate tumorigenesis not only directly from the inside of tumor cells, but also indirectly via immune cells. To explore the immune function of TIPE2 in the development of pancreatic cancer in vivo, we injected Panc02/vector and Panc02/TIPE2 cells into C57BL/6 mice to establish the subcutaneous tumor model. The sizes of tumors were measured weekly one week after tumor cell inoculation. And the results showed that the tumor sizes of TIPE2 group were significantly smaller than vector group during the development of pancreatic cancer (Figure 6A). The mice were sacrificed five weeks after injection of tumor cells and the tumors were isolated, weighted and photographed. The tumor size and weight of TIPE2 group were obviously less than vector group
(Figures 6B, C). The protein levels of TGF $\beta 1$ secreted from cells and expressed in tumor tissues of tumor bearing mice were both detected by ELSIA and IHC, and the expression of TGFBR1 was detected by western blot. Similarly, TIPE2 overexpression could decrease the protein level of TGF $\beta 1$ (Figures 6D, E) and phosphorylation of TGFBR1 (Figure 6F) in pancreatic cancer. Therefore, TIPE2 possibly suppressed the growth of pancreatic cancer through inhibiting TGF $\beta 1$ expression.

\section{TIPE2 Promoted T Cell Activation to Exert Anti-Tumor Effect Through Activation of DCs in Mouse Pancreatic Cancer Model}

To investigate the mechanism of the immune antitumor effect of TIPE2 in pancreatic cancer, we analyzed the tumor-infiltrating lymphocytes via flow cytometry. We found that TIPE2 could increase the percentage of activated $\left(\mathrm{CD} 69^{+}\right)$and effector $\left(\mathrm{CD} 62 \mathrm{~L}^{-} \mathrm{CD} 44^{+}\right) \mathrm{CD}^{+} \mathrm{T}$ and $\mathrm{CD}^{+} \mathrm{T}$ cells (Figure 7A). The percentage of DCs was also increased in TIPE2 group mice. Moreover, the percentage of activated $\mathrm{CD} 80^{+}$DCs was increased, and the percentage of PD-L1 ${ }^{+}$DCs was decreased in TIPE2 group (Figure 7B). How does TIPE2 affect the activation of DCs? Previous study has reported TGF $\beta 1$ could modulate the functions of DCs (22). And we have demonstrated that TIPE2 suppressed the expression of TGF $\beta 1$. Then we co-cultured the Panc02 cells with DCs in vitro. The result showed that TIPE2 also increased the percentage and mean fluorescence intensity of $\mathrm{CD}^{+} 0^{+}$DCs (Figure 7C). Therefore, we inferred that TIPE2 might promote $\mathrm{T}$ cell activation to exert anti-tumor effect through activating DCs in a TGF $\beta 1$ dependent manner.

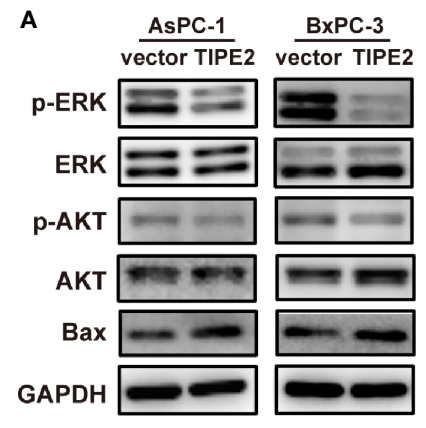

C

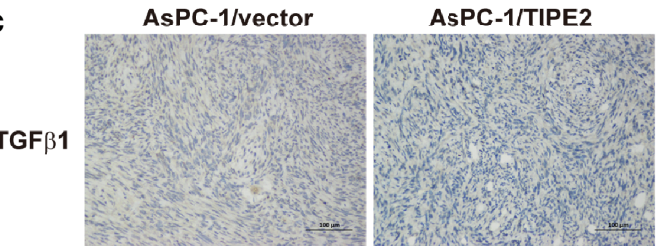

B
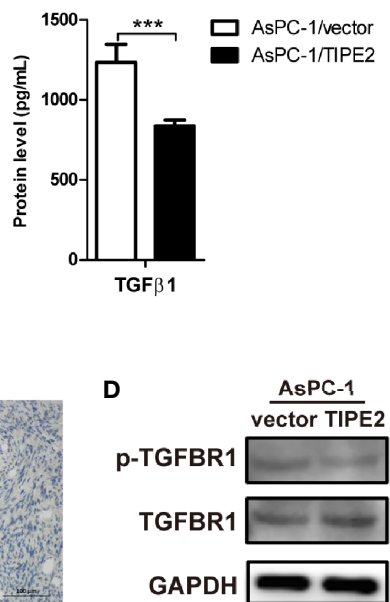

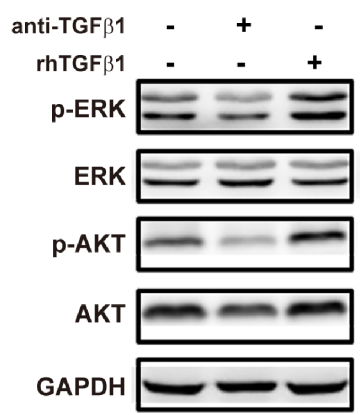

FIGURE 5 | TIPE2 inhibited PI3K/AKT and Raf/MEK/ERK signaling pathways triggered by TGF $\beta 1$. (A) The total proteins extracted from cells were analyzed for the expression of AKT, ERK and Bax using western blot. The antibodies used were anti-pAKT, anti-AKT, anti-pERK, anti-ERK, anti-Bax and anti-GAPDH. (B) ELISA analysis of TGF $\beta 1$ secretion from AsPC-1/vector and AsPC-1/TIPE2 cells. (C) Immunohistochemistry analysis of the TGF 31 expression in AsPC-1/vector and AsPC1/TIPE2 tumor tissues. (D) Western blot analysis of the expression of $\mathrm{p}$-TGFBR1 and total TGFBR1 in AsPC-1/vector and AsPC-1/TIPE2 cells. (E) AsPC-1 cells were seeded in 6-well plate and added with or without anti-TGF $\beta 1$ antibody or rhTGF $\beta 1$ protein. After $48 \mathrm{~h}$ incubation, the total proteins extracted from the cultured AsPC-1 cells were analyzed for the expression of AKT and ERK using western blot. The antibodies used were anti-pAKT, anti-AKT, anti-pERK, anti-ERK and anti$\mathrm{GAPDH}$. Data shown were representative of three independent experiments. Values are presented as means $\pm \mathrm{SD}$. ${ }^{\star \star \star} \mathrm{p}<0.001$. 


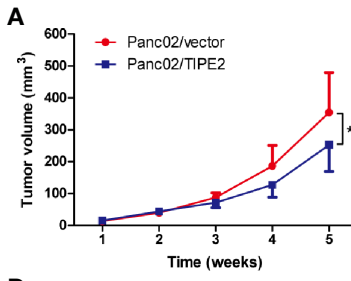

B

C

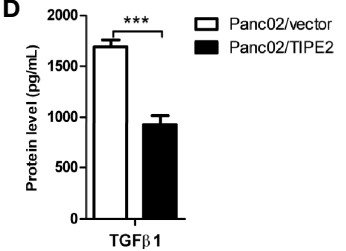

B

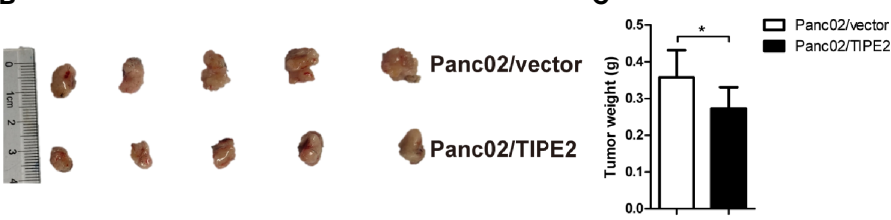

E

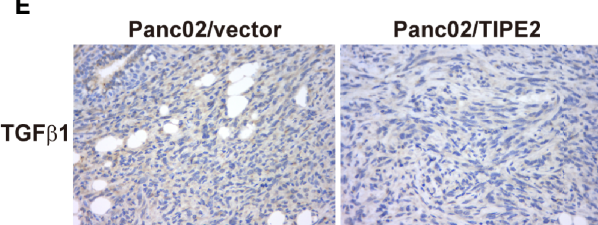

$\mathbf{F}$

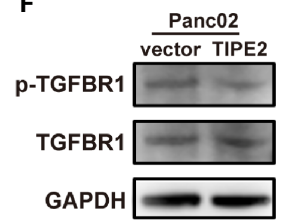

FIGURE 6 | TIPE2 suppressed the growth of pancreatic cancer through inhibiting TGF $\beta 1$ expression in subcutaneous tumor model. (A) The tumor volume was measured weekly one week after tumor inoculation and the tumor growth curve was calculated. (B, C) The tumors were isolated, weighted and photographed five weeks after tumor cells inoculation. (D) ELISA analysis of TGF $\beta 1$ secretion from Panc02/vector and Panc02/TIPE2 cells. (E) Immunohistochemistry analysis of the TGFß1 expression in Panc02/vector and Panc02/TIPE2 tumor tissues. (F) Western blot analysis of the expression of p-TGFBR1 and total TGFBR1 in Panc02/vector and Panc02/TIPE2 cells. The data shown are the representative of three experiments. Values are presented as means \pm SD. ${ }^{*} p<0.05,{ }^{* \star *} p<0.001$.

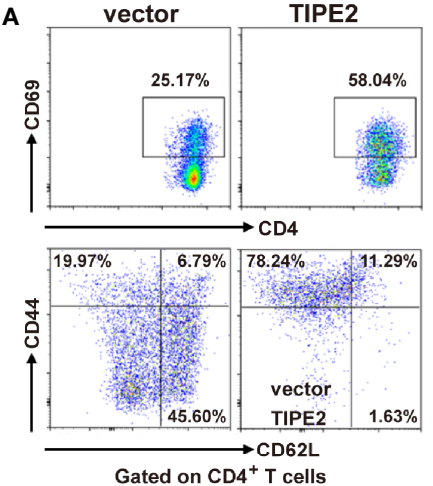

B
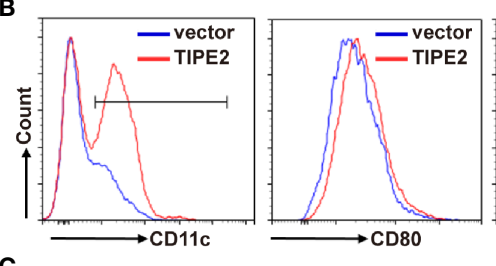

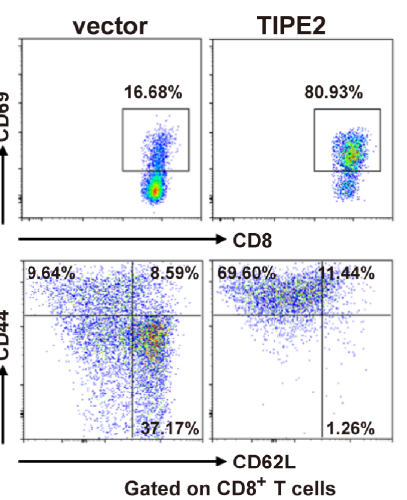

Gated on $\mathrm{CD}^{+} \mathrm{T}$ cells
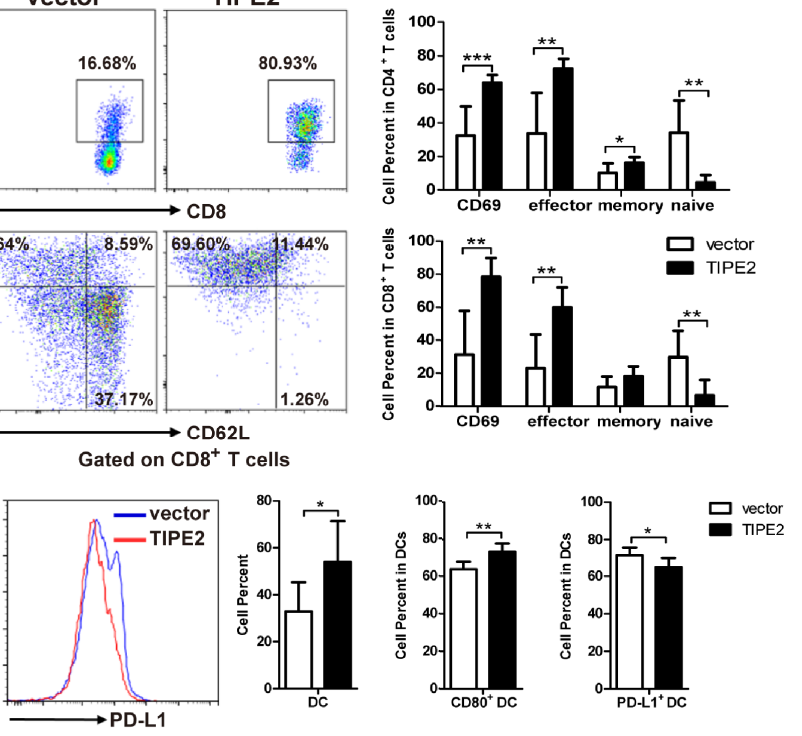

C
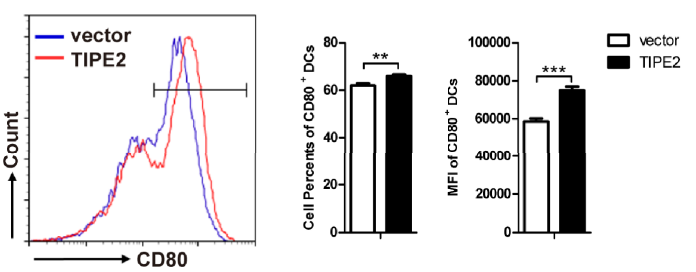

FIGURE 7 | TIPE2 promoted T cell activation to inhibit the development of pancreatic cancer through activation of DCs. (A) Flow cytometry analysis of activated $\left(\mathrm{CD}^{+} 9^{+}\right) \mathrm{CD}^{+} \mathrm{T}$ and $\mathrm{CD} 8^{+} \mathrm{T}$ cells, and the effector $\left(\mathrm{CD} 62 \mathrm{~L}^{-} \mathrm{CD} 44^{+}\right)$of $\mathrm{CD} 4^{+} \mathrm{T}$ and $\mathrm{CD} 8^{+} \mathrm{T}$ cells in TILs. Flow cytometric plots and the summary results were shown. (B) Flow cytometry analysis of DCs, CD80+ DCs and PD-L1+ DCs in tumor-infiltrating lymphocytes. (C) Co-culture DCs and Panc02/vector or Panc02/TIPE2 cells for $24 \mathrm{~h}$. Flow cytometry analysis the activation of DCs (CD80 DCs). Flow cytometric plots and the summary results of percentage and mean fluorescence intensity were shown. The data shown are the representative of three experiments. Values are presented as means \pm SD. ${ }^{*} p<0.05,{ }^{* *} p<0.01,{ }^{* \star *} p<0.001$. 


\section{DISCUSSION}

TIPE2 belongs to TNFAIP8 family which consists of TIPE, TIPE1, TIPE2 and TIPE3. TIPE2 is preferentially expressed in lymphoid tissues and a variety of specific epithelial tissues such as pancreas, thymus, and kidney (23). Preceding studies have established TIPE2 reveals vital roles in inflammation and cancer. In human cancer, TIPE2 is weakly expressed in hepatocellular carcinoma (9), small cell lung cancer (24), gastric cancer (25), breast cancer (26), esophageal cancer (27) and oral tongue squamous cell carcinoma (19), which demonstrated that TIPE2 was involved in cancer progression. In our current study, the expression of TIPE2 was significantly decreased in pancreatic cancer compared to paracancerous tissues, that is consistent with other types of cancers. The expression of TIPE2 was negatively correlated with tumor size in patients. These results indicated that TIPE2 might play an important role in the development of pancreatic cancer.

To further evaluate the regulation role of TIPE2 in the malignancy of pancreatic cancer, we constructed pancreatic cancer cell lines overexpressing TIPE2 or vector control. Overexpression of TIPE2 in pancreatic cancer cells could inhibit cell proliferation, and increase cell apoptosis. Meanwhile, overexpression of TIPE2 suppressed the migration and invasion of pancreatic cancer cells via decreasing the expression of MMPs and $N$-cadherin. As indicated in other studies, both MMPsmediated extracellular matrix degradation/remodeling and $\mathrm{N}$ cadherin were involved in EMT process and metastasis of pancreatic cancer (28-31). Furthermore, TIPE2 indeed reduced the expression of Ki-67 and N-cadherin, increased the expression of Bax, and suppressed the tumor growth of pancreatic cancer in mouse xenograft tumor models.

It has been demonstrated that PI3K/AKT and Raf/MEK/ERK signaling pathways could mediate cell apoptosis, proliferation and metastasis in pancreatic cancer $(28,32-34)$. We demonstrated that TIPE2 could reduce the phosphorylation of ERK and AKT in pancreatic cancer cells. We also found that TIPE2 could upregulate the expression of pro-apoptotic protein Bax, while Bax was involved in the regulation of apoptosis and could influence the prognosis of pancreatic cancer patients (35). As reported, TGF $\beta 1$ is a dual character cytokine during tumorigenesis, and considered to contribute tumor progression by inducing EMT, promoting tumor immune evasion and resisting apoptosis in pancreatic cancer (36). TGF $\beta 1$ triggers Raf/MEK/ERK and PI3K/AKT signaling pathways to promote tumor malignancy in some types of cancers $(20,21)$. TGFBR1 is a key receptor of TGF $\beta 1$, and the phosphorylation of TGFBR1 is involved in these processes $(37,38)$. Indeed, we found that blocking TGF $\beta 1$ with anti-TGF $\beta 1$ antibody could reduce the phosphorylation of ERK and AKT in pancreatic cancer, while treating with TGF $\beta 1$ protein showed the opposed effect. Furthermore, TIPE2 could not only inhibit the secretion of TGF $\beta 1$, but also decrease the phosphorylation of TGFBR1 in pancreatic cancer cells. These results suggested that TIPE2 might affect pancreatic cancer via inhibiting PI3K/AKT and Raf/MEK/ ERK signaling pathways triggered by TGF $\beta 1$.

TIPE2, as a crucial regulator in immune responses and homeostasis, involved in both innate and adaptive immune responses, can regulate tumorigenesis not only directly from the inside of tumor cells, but also indirectly via immune cells (39). To explore the immune-regulation role of TIPE2 in pancreatic cancer, we established the subcutaneous tumor model through injecting mouse Panc02/vector and Panc02/ TIPE2 cells into mice. TGF $\beta 1$ is a main factor inducing immunosuppression in tumor microenvironment of pancreatic cancer, and inhibition of TGF $\beta 1$ strategy may provide a new opportunity for the treatment of pancreatic cancer (36). Therefore, we speculated that TIPE2 may play an anti-tumor role through inhibiting TGF $\beta 1$ in tumor microenvironment. Indeed, TIPE2 inhibited the growth of pancreatic cancer in mice subcutaneous tumor model and the expression of TGF $\beta 1$ in tumor cells was suppressed. Furthermore, we found that TIPE2 could promote T cell and DC activation to inhibit the development of pancreatic cancer. The upregulating of costimulatory molecules on DCs which interact with CD28 receptor molecules on the $\mathrm{T}$ cells could promote $\mathrm{T}$ cell activation $(40,41)$. TIPE2 also upregulated the expression of CD80 on DCs. Therefore, TIPE2 may activate T cells through promoting the activation of DCs. Then we found that TIPE2 could decrease the cell percentage of PD-L1 ${ }^{+}$DCs in tumor-infiltrating lymphocytes. According to the report that PD-L1 expression on DCs could attenuate $\mathrm{T}$ cell activation recently (42), we speculated that TIPE2 could activate T cells not only through activating DCs, but also through inhibiting PD-L1 expression on DCs. But why TIPE2 could activate DCs and inhibit PD-L1 expression on DCs? TGF $\beta 1$, as one of the most important factors of modulating the function of DCs in tumor microenvironment, has been proved that could induce DCs tolerance directly, or increase the expression of PD-L1 on DCs in a STAT3 dependent manner $(22,43)$. Additionally, we also found that TIPE2 could activate DCs in vitro. Therefore, TIPE2 might exert an anti-tumor effect by activation $\mathrm{T}$ cells through DCs in a TGF $\beta 1$ dependent manner in pancreatic cancer. However, the regulation mechanism of TGF $\beta 1$ regulated by TIPE2 is not clear, which need to be further investigated.

Taken together, our present study demonstrated that the expression of TIPE2 was reduced in human pancreatic cancer, which was negatively correlated with tumor size. Overexpression of TIPE2 significantly suppressed the proliferation, metastasis, and promoted apoptosis of pancreatic cancer possibly through inhibition of PI3K/AKT and Raf/MEK/ERK signaling pathways triggered by TGF $\beta 1$. Moreover, TIPE2 promoted $\mathrm{T}$ cell activation to exert an anti-tumor effect possibly through activation of DCs in a TGF $\beta 1$ dependent manner. In summary, we described the multiple regulatory mechanisms of TIPE2 in pancreatic tumorigenesis and tumor microenvironment, based on the result obtained it is suggested that TIPE2 may serve as a potential therapeutic target in pancreatic cancer.

\section{DATA AVAILABILITY STATEMENT}

The original contributions presented in the study are included in the article/Supplementary Material. Further inquiries can be directed to the corresponding authors. 


\section{ETHICS STATEMENT}

The animal study was reviewed and approved by the Institutional Laboratory Animal Care and Use Committee of Soochow University.

\section{AUTHOR CONTRIBUTIONS}

ZZ, FF and CL conceived and designed the study. FF, CL, HB, YZ and LZ performed the experiments. FF, CL and WC analysed the data. ZZ, FF and CL wrote and revised the manuscript. All authors contributed to the article and approved the submitted version.

\section{REFERENCES}

1. Siegel RL, Miller KD, Jemal A. Cancer Statistics, 2019. CA Cancer J Clin (2019) 69(1):7-34. doi: 10.3322/caac.21551

2. Rahib L, Smith BD, Aizenberg R, Rosenzweig AB, Fleshman JM, Matrisian LM. Projecting Cancer Incidence and Deaths to 2030: The Unexpected Burden of Thyroid, Liver, and Pancreas Cancers in the United States. Cancer Res (2014) 74(11):2913-21. doi: 10.1158/0008-5472.CAN-14-0155

3. Teague A, Lim KH, Wang-Gillam A. Advanced Pancreatic Adenocarcinoma: A Review of Current Treatment Strategies and Developing Therapies. Ther Adv Med Oncol (2015) 7(2):68-84. doi: 10.1177/1758834014564775

4. Zhan HX, Xu JW, Wu D, Wu ZY, Wang L, Hu SY, et al. Neoadjuvant Therapy in Pancreatic Cancer: A Systematic Review and Meta-Analysis of Prospective Studies. Cancer Med (2017) 6(6):1201-19. doi: 10.1002/cam4.1071

5. Balachandran VP, Beatty GL, Dougan SK. Broadening the Impact of Immunotherapy to Pancreatic Cancer: Challenges and Opportunities. Gastroenterology (2019) 156(7):2056-72. doi: 10.1053/j.gastro.2018.12.038

6. Schizas D, Charalampakis N, Kole C, Economopoulou P, Koustas E, Gkotsis E, et al. Immunotherapy for Pancreatic Cancer: A 2020 Update. Cancer Treat $\operatorname{Rev}(2020)$ 86:102016. doi: 10.1016/j.ctrv.2020.102016

7. Zambirinis CP, Miller G. Cancer Manipulation of Host Physiology: Lessons From Pancreatic Cancer. Trends Mol Med (2017) 23(5):465-81. doi: 10.1016/ j.molmed.2017.03.003

8. Leinwand J, Miller G. Regulation and Modulation of Antitumor Immunity in Pancreatic Cancer. Nat Immunol (2020) 21(10):1152-9. doi: 10.1038/s41590020-0761-y

9. Gus-Brautbar Y, Johnson D, Zhang L, Sun H, Wang P, Zhang S, et al. The Anti-Inflammatory TIPE2 Is an Inhibitor of the Oncogenic Ras. Mol Cell (2012) 45(5):610-8. doi: 10.1016/j.molcel.2012.01.006

10. Sun H, Gong S, Carmody RJ, Hilliard A, Li L, Sun J, et al. TIPE2, A Negative Regulator of Innate and Adaptive Immunity That Maintains Immune Homeostasis. Cell (2008) 133(3):415-26. doi: 10.1016/j.cell.2008.03.026

11. Cao X, Zhang L, Shi Y, Sun Y, Dai S, Guo C, et al. Human Tumor Necrosis Factor (TNF)-Alpha-Induced Protein 8-Like 2 Suppresses Hepatocellular Carcinoma Metastasis Through Inhibiting Rac1. Mol Cancer (2013) 12 (1):149. doi: 10.1186/1476-4598-12-149

12. Li Z, Zhang W, Li Y, Cao S, Liu S, Ning L, et al. TIPE2 Acts as a Biomarker for GIST Risk Category and Suppresses the Viability and Invasiveness of GIST Cells. Cell Biosci (2018) 8:62. doi: 10.1186/s13578-018-0261-Z

13. Zhang YH, Yan HQ, Wang F, Wang YY, Jiang YN, Wang YN, et al. TIPE2 Inhibits TNF-Alpha-Induced Hepatocellular Carcinoma Cell Metastasis Via Erk1/2 Downregulation and NF-kappaB Activation. Int J Oncol (2015) 46 (1):254-64. doi: 10.3892/ijo.2014.2725

14. Liu ZJ, Liu HL, Zhou HC, Wang GC. Tipe2 Inhibits Hypoxia-Induced Wnt/ Beta-Catenin Pathway Activation and EMT in Glioma Cells. Oncol Res (2016) 24(4):255-61. doi: 10.3727/096504016X14666990347356

15. Wu J, Zhang H, Xu C, Xu H, Zhou X, Xie Y, et al. TIPE2 Functions as a Metastasis Suppressor Via Negatively Regulating Beta-Catenin Through Activating GSK3beta in Gastric Cancer. Int J Oncol (2016) 48(1):199-206. doi: $10.3892 /$ ijo.2015.3224

16. Wang K, Ren Y, Liu Y, Zhang J, He JJ. Tumor Necrosis Factor (TNF)-AlphaInduced Protein 8-Like-2 (TIPE2) Inhibits Proliferation and Tumorigenesis in

\section{FUNDING}

This work was supported by National Natural Science Foundation of China (NSFC, 81903248) and the project funding from Suzhou Ninth People's Hospital (YK202036).

\section{SUPPLEMENTARY MATERIAL}

The Supplementary Material for this article can be found online at: https://www.frontiersin.org/articles/10.3389/fonc.2021. 680985/full\#supplementary-material

Breast Cancer Cells. Oncol Res (2017) 25(1):55-63. doi: 10.3727/096504016X 14719078133320

17. Liu Y, Wang X, Wan L, Liu X, Yu H, Zhang D, et al. TIPE2 Inhibits the Migration and Invasion of Endometrial Cells by Targeting Beta-Catenin to Reverse Epithelial-Mesenchymal Transition. Hum Reprod (2020) 35(6):137790. doi: 10.1093/humrep/deaa062

18. Zhang Z, Liu L, Cao S, Zhu Y, Mei Q. Gene Delivery of TIPE2 Inhibits Breast Cancer Development and Metastasis Via CD8(+) T and NK Cell-Mediated Antitumor Responses. Mol Immunol (2017) 85:230-7. doi: 10.1016/ j.molimm.2017.03.007

19. Zhao LL. TIPE2 Suppresses Progression and Tumorigenesis of the Oral Tongue Squamous Cell Carcinoma by Regulating FoxP3(+) Regulatory T Cells. J Bioenerg Biomembr (2020) 52(4):279-89. doi: 10.1007/s10863-02009840-w

20. Rodriguez-Garcia A, Samso P, Fontova P, Simon-Molas H, Manzano A, Castano E, et al. TGF-Betal Targets Smad, P38 MAPK, and PI3K/Akt Signaling Pathways to Induce PFKFB3 Gene Expression and Glycolysis in Glioblastoma Cells. FEBS J (2017) 284(20):3437-54. doi: 10.1111/febs.14201

21. Ellenrieder V, Hendler SF, Boeck W, Seufferlein T, Menke A, Ruhland C, et al. Transforming Growth Factor Betal Treatment Leads to an EpithelialMesenchymal Transdifferentiation of Pancreatic Cancer Cells Requiring Extracellular Signal-Regulated Kinase 2 Activation. Cancer Res (2001) 61 (10):4222-8.

22. Esebanmen GE, Langridge WHR. The Role of TGF-Beta Signaling in Dendritic Cell Tolerance. Immunol Res (2017) 65(5):987-94. doi: 10.1007/ s12026-017-8944-9

23. Li T, Wang W, Gong S, Sun H, Zhang H, Yang AG, et al. Genome-Wide Analysis Reveals TNFAIP8L2 as an Immune Checkpoint Regulator of Inflammation and Metabolism. Mol Immunol (2018) 99:154-62. doi: 10.1016/j.molimm.2018.05.007

24. Liu QQ, Zhang FF, Wang F, Qiu JH, Luo CH, Zhu GY, et al. TIPE2 Inhibits Lung Cancer Growth Attributing to Promotion of Apoptosis by Regulating Some Apoptotic Molecules Expression. PloS One (2015) 10(5):e0126176. doi: 10.1371/journal.pone.0126176

25. Zhao Q, Zhao M, Dong T, Zhou C, Peng Y, Zhou X, et al. Tumor Necrosis Factor-Alpha-Induced Protein-8 Like-2 (TIPE2) Upregulates p27 to Decrease Gastic Cancer Cell Proliferation. J Cell Biochem (2015) 116(6):1121-9. doi: $10.1002 /$ jcb. 25068

26. Zhang Z, Liu L, Liu C, Cao S, Zhu Y, Mei Q. TIPE2 Suppresses the Tumorigenesis, Growth and Metastasis of Breast Cancer Via Inhibition of the AKT and p38 Signaling Pathways. Oncol Rep (2016) 36(6):3311-6. doi: 10.3892/or.2016.5192

27. Zhu L, Zhang X, Fu X, Li Z, Sun Z, Wu J, et al. TIPE2 Suppresses Progression and Tumorigenesis of Esophageal Carcinoma Via Inhibition of the Wnt/BetaCatenin Pathway. J Transl Med (2018) 16(1):7. doi: 10.1186/s12967-0181383-0

28. Ding Q, Miyazaki Y, Tsukasa K, Matsubara S, Yoshimitsu M, Takao S. CD133 Facilitates Epithelial-Mesenchymal Transition Through Interaction With the ERK Pathway in Pancreatic Cancer Metastasis. Mol Cancer (2014) 13:15. doi: $10.1186 / 1476-4598-13-15$

29. He Y, Liu XD, Chen ZY, Zhu J, Xiong Y, Li K, et al. Interaction Between Cancer Cells and Stromal Fibroblasts Is Required for Activation of the 
uPAR-uPA-MMP-2 Cascade in Pancreatic Cancer Metastasis. Clin Cancer Res (2007) 13(11):3115-24. doi: 10.1158/1078-0432.CCR-06-2088

30. Nakajima S, Doi R, Toyoda E, Tsuji S, Wada M, Koizumi M, et al. N-Cadherin Expression and Epithelial-Mesenchymal Transition in Pancreatic Carcinoma. Clin Cancer Res (2004) 10(12 Pt 1):4125-33. doi: 10.1158/1078-0432.CCR-0578-03

31. Ogawa K, Lin Q, Li L, Bai X, Chen X, Chen H, et al. Aspartate BetaHydroxylase Promotes Pancreatic Ductal Adenocarcinoma Metastasis Through Activation of SRC Signaling Pathway. J Hematol Oncol (2019) 12 (1):144. doi: 10.1186/s13045-019-0837-Z

32. Ji S, Qin Y, Shi S, Liu X, Hu H, Zhou H, et al. ERK Kinase Phosphorylates and Destabilizes the Tumor Suppressor FBW7 in Pancreatic Cancer. Cell Res (2015) 25(5):561-73. doi: 10.1038/cr.2015.30

33. Meng Q, Shi S, Liang C, Liang D, Hua J, Zhang B, et al. Abrogation of Glutathione Peroxidase-1 Drives EMT and Chemoresistance in Pancreatic Cancer by Activating ROS-Mediated Akt/GSK3beta/Snail Signaling. Oncogene (2018) 37(44):5843-57. doi: 10.1038/s41388-018-0392-z

34. Ripka S, Neesse A, Riedel J, Bug E, Aigner A, Poulsom R, et al. CUX1: Target of Akt Signalling and Mediator of Resistance to Apoptosis in Pancreatic Cancer. Gut (2010) 59(8):1101-10. doi: 10.1136/gut.2009.189720

35. Friess H, Lu Z, Graber HU, Zimmermann A, Adler G, Korc M, et al. Bax, But Not bcl-2, Influences the Prognosis of Human Pancreatic Cancer. Gut (1998) 43(3):414-21. doi: 10.1136/gut.43.3.414

36. Batlle E, Massague J. Transforming Growth Factor-Beta Signaling in Immunity and Cancer. Immunity (2019) 50(4):924-40. doi: 10.1016/j.immuni.2019.03.024

37. Lee MK, Pardoux C, Hall MC, Lee PS, Warburton D, Qing J, et al. TGF-Beta Activates Erk MAP Kinase Signalling Through Direct Phosphorylation of Shca. EMBO J (2007) 26(17):3957-67. doi: 10.1038/sj.emboj.7601818

38. Zhang L, Zhou F, ten Dijke P. Signaling Interplay Between Transforming Growth Factor-Beta Receptor and PI3K/AKT Pathways in Cancer. Trends Biochem Sci (2013) 38(12):612-20. doi: 10.1016/j.tibs.2013.10.001
39. Yan D, Wang J, Sun H, Zamani A, Zhang H, Chen W, et al. TIPE2 Specifies the Functional Polarization of Myeloid-Derived Suppressor Cells During Tumorigenesis. J Exp Med (2020) 217(2):e20182005. doi: 10.1084/ jem.20182005

40. Kalinski P, Hilkens CM, Wierenga EA, Kapsenberg ML. T-Cell Priming by Type- 1 and Type-2 Polarized Dendritic Cells: The Concept of a Third Signal. Immunol Today (1999) 20(12):561-7. doi: 10.1016/s0167-5699(99)01547-9

41. Randolph GJ, Ochando J, Partida-Sanchez S. Migration of Dendritic Cell Subsets and Their Precursors. Annu Rev Immunol (2008) 26:293-316 doi: 10.1146/annurev.immunol.26.021607.090254

42. Peng Q, Qiu X, Zhang Z, Zhang S, Zhang Y, Liang Y, et al. PD-L1 on Dendritic Cells Attenuates $\mathrm{T}$ Cell Activation and Regulates Response to Immune Checkpoint Blockade. Nat Commun (2020) 11(1):4835. doi: 10.1038/ s41467-020-18570-x

43. Song S, Yuan P, Wu H, Chen J, Fu J, Li P, et al. Dendritic Cells With an Increased PD-L1 by TGF-Beta Induce T Cell Anergy for the Cytotoxicity of Hepatocellular Carcinoma Cells. Int Immunopharmacol (2014) 20(1):117-23. doi: 10.1016/j.intimp.2014.02.027

Conflict of Interest: The authors declare that the research was conducted in the absence of any commercial or financial relationships that could be construed as a potential conflict of interest.

Copyright (c) 2021 Feng, Liu, Bian, Cai, Zhou, Zhou and Zhuang. This is an openaccess article distributed under the terms of the Creative Commons Attribution License (CC BY). The use, distribution or reproduction in other forums is permitted, provided the original author(s) and the copyright owner(s) are credited and that the original publication in this journal is cited, in accordance with accepted academic practice. No use, distribution or reproduction is permitted which does not comply with these terms. 